\title{
Technique to Calculate the Strength of the Combined Gas Cylinder High Pressure
}

\author{
Emad Toma Bane Karash \\ Technical of Education, Mosul Institute, Mosul, Iraq
}

Email address:

emadbane2007@yahoo.com

\section{To cite this article:}

Emad Toma Bane Karash. Technique to Calculate the Strength of the Combined Gas Cylinder High Pressure. Advances in Materials. Vol. 4, No. 2, 2015, pp. 36-42. doi: 10.11648/j.am.20150402.13

\begin{abstract}
Two types of composite cylindrical high-pressure vessels load-carrying capacity design procedure based on the discrete - structural theory are considered in this paper. The new design of the combined cylindrical vessel is offered. The cylinder includes an internal bearing fiberglass shell and an external protective metal cover. The vacuity between a fiberglass shell and a covering is filled with a soft material that allows regulating contact pressure which is transferred from a fiberglass shell to a metal covering.
\end{abstract}

Keywords: Vessel, Multilayered Shells, Cylinder, Composite Materials, Fiberglass Shell

\section{Introduction}

Cylindrical pressure vessels are widely used for commercial, under water vehicles and in aerospace applications. At present the outer shells of the pressure vessels are made up of conventional metals like steels and aluminum alloys. The payload performance/ speed/ operating range depends upon the weight. The lower the weight the better the performance, one way of reducing the weight is by reducing the weight of the shell structure. The use of composite materials improves the performance of the vessel and offers a significant amount of material savings. Moreover, the stacking sequence is very crucial to the strength of the composite material. This Project involves various objective functions such as stiffness, buckling load and Weight at each level of optimization. Usually composite pressure vessels are designed for minimum mass under strength constraints. A graphical analysis is presented to find optimum fiber orientation for given layer thicknesses.

Due to the limited sources available most of the oil world vehicle manufacturers. Have always shown and are interested in research on the use of alternative motor fuels - natural gas Biofuels hydrogen etc. Extensive use of natural gas due to the fact that its production does not require a deep chemical processing virgin materials and training to use about lead by physical methods such as: compressed or liquefied. Because of this. In most countries the cost of gas motor fuels to consumers below the cost of liquid. Translation to gas does not require constructive alteration of the engine: enough to put gas equipment. Increases the reliability of the fuel system of the car, as the gas installation duplicates the original gasoline.

In recent years, road transport many countries rising rates of use of liquefied natural gas (LNG) as a motor fuel. Ukraine is also interested in retrofitting vehicles to run on natural gas. This is facilitated by gas transmission system development, the availability of resources of natural gas and coal mine methane a network of existing CNG filling stations by the number of CNG vehicles. Ukraine ranks ninth in the world and is a leader among the CIS countries in the use of alternative fuels.

When the supply of gas in steel cylinders that are widely used at the present time the volume of gas supplied is limited carrying capacity of the vehicle. The use of lightweight composite cylinders will increase the volume of gas to be transported and reducing the cost of the container leads to a decrease in the cost of gas transportation.

Classification of cylindrical pressure vessels for storage of natural gas gives the international standard ISO 11439-2003 [1] and GOST R 51753-2001. [2] The main advantage of cylinders made of composite material - a lower mass. Compared with all-cylinder unit weight of the composite material is reduced by 2-3 times [3]. However, due to the high cost of materials and labor intensive composite manufacturing cost technology such cylinders above.

For fuel supply one internal combustion engine is required 
from 2-3 cylinders for cars up to 10 or more for trucks tractors or locomotives. Dimensions of existing vehicles to limit the size of the cylinders. In addition, gas cylinders significantly reduce the carrying capacity of vehicles and require the high cost of fuel for their own transportation than they massive. However, they operate in a cyclic thermo mechanical loads.

In this regard, the creation of new structures of gas cylinders simple and high-tech methods of modern materials still remains an urgent task. The most obvious way to improve - reducing the amount of metal in the construction of the container by replacing it with alternative materials with the best design characteristics.

Plastic high pressure cylinders with reinforcing shell on the cylindrical part - the next step to solving the problem of production cost-effective, simple to manufacture and reliable pressure vessels. This problem is solved by the use of combined cylinders in which the sealed metal shell combined with the power envelope of composite materials. In addition, the design of the cylinder may impose additional layers of the protective and other functions. Thus the compounds of the balloon layers with different functional purpose.

Composite pressure vessels are designed for minimum mass under strength constraints. A graphical analysis is presented to find optimum fiber orientation for given layer thicknesses. In the present work, an analytical model is developed for the Prediction of the minimum buckling load with / without stiffener composite shell of continuous angle ply laminas $\left( \pm 45^{\circ}, \pm 55^{\circ}, \pm 65^{\circ}, \pm 75^{\circ}, \pm 85^{\circ}\right)$ for investigation. Comparisons are made for two different approaches i.e. the finite element model and the theoretical model. A 3-D finite element analysis is built using ANSYS-12.0 version software into consideration, for static and buckling analysis on the pressure vessel [4-7]
In accordance with the international standard ISO 114392003 [1] and GOST R 51753-2001 [2].The design pressure of the destruction of the cylinders manufactured using polymer composite materials shall be determined on the basis of analysis of stresses in the fiber. The requirement for safety factor cylinders increases in the series: carbon fiber organic fiber glass fiber. For cylinders based on organic and glass fibers safety margin requirement increases with the proportion in the composite structure. Only cylinders based on carbon fibers requirement for safety margin almost constant for all types of structures including all-metal cylinders. Design pressure cylinders destruction of all types should be at least 2.6 F (P - working pressure).

In the design of composite structures takes place quite a number of possible options and schemes of reinforcement. Therefore, the theoretical problem of determining the optimal deformation and strength properties of such materials at the lowest cost to the experiment seemed to be relevant.

In the composite material with a regular structure usually present in the form of repetitive elements unidirectional layers.

Neglecting the heterogeneity of structure at the micro level of each layer. We can find effective characteristics of the individual layers at the macro level. In this case, the material deformation model is quasi-homogeneous structure composed of different layers.

\section{Basic Equations and Geometric Equations}

For a rotating shell which comprises $\mathrm{n}$ layers of coaxial surfaces of revolution.in [8] a resolving system of partial differential equations of the form

$$
\frac{d \vec{Y}^{(k)}}{A_{(k)} d \alpha_{1}}=D_{\circ}^{(k)} \vec{Y}^{(k)}+D_{1}^{(k)} \frac{\partial \vec{Y}^{(k)}}{B_{(k)} \partial \alpha_{2}^{(k)}}+D_{2}^{(k)} \frac{\partial^{2} \vec{Y}^{(k)}}{B_{(k)}^{2} \partial \alpha_{2}^{(k) 2}}+\vec{f}^{(k)}, \quad k=1,2, \ldots, n
$$

Where

$$
\vec{Y}^{(k)}=\left\{T_{11}^{(k)}, T_{12}^{(k)}, Q_{1}^{(k)}, M_{11}^{(k)}, M_{12}^{(k)}, L_{11}^{(k)}, L_{12}^{(k)}, u_{1}^{(k)}, u_{2}^{(k)}, w^{(k)}, \gamma_{1}^{(k)}, \gamma_{2}^{(k)}, \psi_{1}^{(k)}, \psi_{2}^{(k)}\right\}^{T}, \vec{f}^{(k)}=\left\{f_{1}^{(k)}, f_{2}^{(k)}, \ldots, f_{14}^{(k)}\right\}, D_{\circ}^{(k)}, D_{1}^{(k)}, D_{2}^{(k)}-
$$

14 square matrices of order $n$.

The main unknown function takes the values that define the boundary conditions on the contour $1(\mathrm{k})$. Kinematic and static conditions perfect contact of the individual layers of thin-walled elements on personal mating surfaces can be written:

$$
\begin{gathered}
2 u_{i}^{(2)}=u_{i}^{(k+1)}+u_{i}^{(k-1)}-\frac{h^{(k+)}}{2} \gamma^{(k+1)}+\frac{h^{(k-1)}}{2} \gamma_{i}^{(k-1)}-\phi^{(k+1)}\left(\frac{h^{(k+1)}}{2}\right) \psi_{i}^{(k+1)}+\phi^{(k-1)}\left(\frac{h^{(k-1)}}{2}\right) \psi_{i}^{(k-1)}, \quad(i=1,2) \\
2 w^{(k)}=w^{(k+1)}+w^{(k-1)}-\frac{h^{(k+)}}{2} \gamma^{(k+1)}+\frac{h^{(k-1)}}{2} \gamma_{i}^{(k-1)}-\frac{h^{(k+1)}}{2} \gamma^{(k-1)} \\
\sigma_{i 3}^{(k)+}=\sigma_{i 3}^{(k+1)-}, \sigma_{i 3}^{(k)-}=\sigma_{i 3}^{(k-1)+} \quad(i=1,2) \\
\sigma_{33}^{(k)+}=\sigma_{33}^{(k+1)-} \quad, \quad \sigma_{33}^{(k) 1}=\sigma_{33}^{(k-1)+} .
\end{gathered}
$$

Performing kinematic (2) and static (3) the conditions on personal contact mating surfaces using the penalty function method [8]. Are the entire systems of governing equations (1) to solve the contact problem of discrete-continuum theory of multilayer shells.

The decision provided the problem is obtained by the 
method of orthogonal sweep S.K.Godunova [9]. Establish unambiguous relationship efforts between the edge at the ends of the $m$-th shell element and boundary movements of these ends. On the basis of movement equates balance for each node element and a system of linear algebraic equations for the generalized displacements of nodes multilayer shell structure. When moving nodes structure known from the solution of the inverse problem of the method is determined by the movement of the stress-strain state of the overall structure.

To avoid contact fiberglass shell with a metal shell provides a gap that is filled with an isotropic material of low density, for example, penovinilplastom with the following technical parameters: $E=83 M P a ; v=0.33$. Model of the combined high-pressure cylinder is shown in Fig. 1.

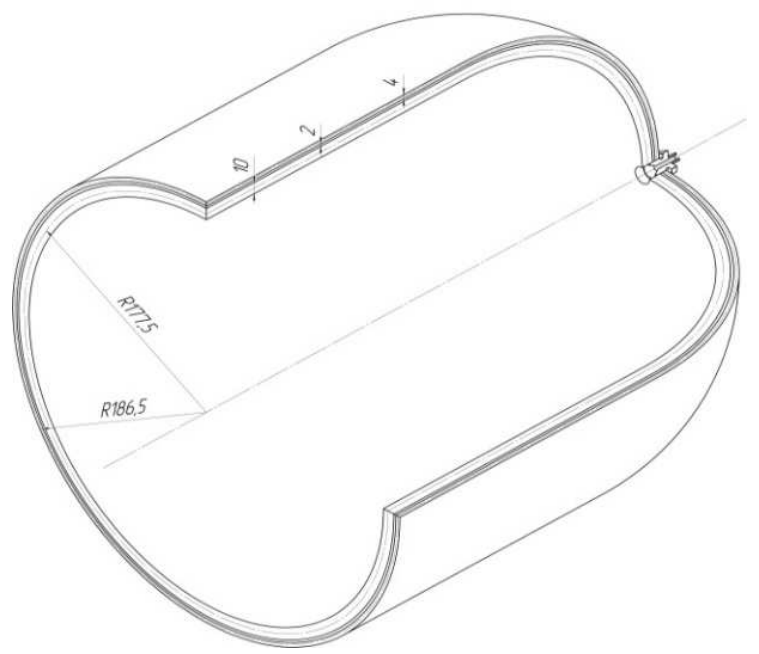

Fig. 1. The analytical model of the combined high-pressure cylinder.

Model of the combined high-pressure cylinder is shown in Fig. 2. In general, the balloon consists of two parts: the inner part - fiberglass exterior - metal.

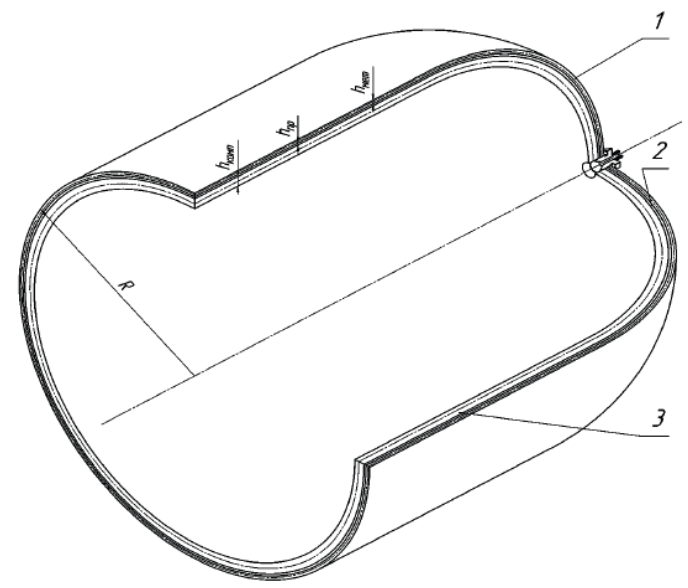

Fig. 2. The analytical model of the combined high-pressure cylinder: 1 Protective metal shell (duralumin material V-95); 2 - regulating pliable layer; 3 - bearing fiberglass shell.

In the space of the parameters of the stress-strain state of the composite of any physically permissible limit state criteria defines a closed convex surface [10]. This tensorpolynomial criterion is expressed as follows [11]:

$$
\left(R_{i j} \sigma_{i j}\right)^{2}+\left(R_{i j k l} \sigma_{i j} \sigma_{k l}\right)^{\beta}+\left(R_{i j k l m n} \sigma_{i j} \sigma_{k l} \sigma_{m n}\right)^{\gamma}+\ldots=1
$$

Where

$\sigma_{1}=\sigma_{11}, \sigma_{2}=\sigma_{22}, \sigma_{3}=\sigma_{33}, \sigma_{4}=\sigma_{23}, \sigma_{5}=\sigma_{13}, \sigma_{6}=\sigma_{12} ;$ $R_{i j}, R_{i j k l}, R_{i j k l m n}$ - matrix notation tensor surface strength of the second, fourth, sixth and following even rank. coefficients $R_{i j k}, R_{i j}, R_{i j k}$ and exponents $\alpha, \beta, \gamma$ determined to give the best description of the experimental data. Is there $R_{i}$ is a second-rank tensor: $R_{i} \sigma_{i}=\tilde{R}_{i k} \sigma_{i k}$, - has six independent components. Similarly, $R_{i j} \sigma_{i} \sigma_{j}=\tilde{R}_{i j k} \sigma_{i k} \sigma_{j k}$ contains 21 independent components. All higher-order tensors are the most general form. With the help of the exponents is not reached, no enhancement of community; almost always believe $\alpha=\beta=\gamma=1$. Tensors $R_{i j k}, R_{i j}, R_{i j k}$ simplified symmetry conditions of the composite material.

In engineering practice, more convenient in terms of practical application proved the following criterion strength tensor-polynomial form:

$$
R_{i j} \sigma_{i j}+R_{i j k l} \sigma_{i j} \sigma_{k l}+R_{i j k l m n} \sigma_{i j} \sigma_{k l} \sigma_{m n}+\ldots=1,(i, j, k, m, l, n=1,2,3),
$$

Which is easily obtained from (1), taking $\alpha, \beta, \gamma, \ldots=1$.

Most of the known polynomial strength criteria are, as a rule, a particular case of the criterion (5).

Using strength criterion (2) in the form

$$
R_{i j} \sigma_{i j}+R_{i j k l} \sigma_{i j} \sigma_{k l}+R_{i j k l m n} \sigma_{i j} \sigma_{k l} \sigma_{m n}=1,(i, j, k, m, l, n=1,2,3),
$$

You can examine the conditions fracture in a layered composite as a whole. Assumptions about the independence of the loading path, on a linear elastic behavior of the material and the absence of interlayer interactions reduced the number of strength tensor in equation (6) for an orthotropic composite with plane stress to ten. Layered composite strength criterion (6), for the practical application proved to be quite difficult, as it implies conduct complex experiments to find the coefficients of the tensors surface strength.

In most cases, the destruction of the composite laminate begins with the destruction of a single layer or linkages between them. Therefore, the construction of the limit surface is considered that the destruction is localized in a single layer and strength criterion should be exactly for this layer.

Approximation of the limit surface strength of orthotropic layer second-degree polynomial is considered in [12]. Equation (6) takes the form

$$
R_{i j} \sigma_{i j}+R_{i j k l} \sigma_{i j} \sigma_{k l}=1,(i, j, k, l=1,2,3),
$$

Where $R_{i j}, R_{i j k l}$ - surface layer strength tensors of second and fourth order.

In the case of plane stress equation (7) represents the 
marginal surface (ellipsoid) in three-dimensional stress space:

$$
R_{11} \sigma_{11}+R_{22} \sigma_{22}+2 R_{12} \sigma_{12}+R_{1111} \sigma_{11}^{2}+R_{2222} \sigma_{22}^{2}+4 R_{1212} \sigma_{12}^{2}+2 R_{1122} \sigma_{11} \sigma_{22}++4 R_{1112} \sigma_{11} \sigma_{12}+4 R_{2212} \sigma_{22} \sigma_{12}=1 .
$$

The coefficients of (5) are determined using the experimentally determined limit of strength $\sigma_{i j}^{+}, \sigma_{i j}^{-}$ $(i, j=1,2)$. The index of "+" means that this component - the

ultimate tensile stress index "-" denotes he ultimate stress in compression. Tensor components for surface strength (8) in [12] proposed the following relationship:

$$
\begin{gathered}
R_{11}=\frac{\sigma_{11}^{-}-\sigma_{11}^{+}}{\sigma_{11}^{-} \sigma_{11}^{+}} ; R_{22}=\frac{\sigma_{22}^{-}-\sigma_{22}^{+}}{\sigma_{22}^{-} \sigma_{22}^{+}} ; R_{12}=\frac{\sigma_{12}^{-}-\sigma_{12}^{+}}{\sigma_{12}^{-} \sigma_{12}^{+}} ; R_{1111}=\frac{1}{\sigma_{11}^{-} \sigma_{11}^{+}} ; R_{2222}=\frac{1}{\sigma_{22}^{-} \sigma_{22}^{+}} ; 4 R_{1212}=\frac{1}{\sigma_{12}^{-} \sigma_{12}^{+}} ; \\
2 R_{1122}=\frac{R_{11}-R_{22}}{\sigma_{12}^{-}}+R_{1111}+R_{2222}+\frac{1}{\left(\sigma_{12}^{-}\right)^{2}} .
\end{gathered}
$$

In (8) - (9) tensors into account the possible difference in strength characteristics of the material strength in tension and compression.

It should be noted that the strength of the material does not depend on the sign of the tangential stress limit value, i.e. $\sigma_{12}^{-}=\sigma_{12}^{+}$. In addition, for an orthotropic material symmetry axes true identity $-R_{1112}=R_{2212}=0$. Available experimental values $\sigma_{i j}^{+}, \sigma_{i j}^{-}(i, j=1,2)$ not enough to determine the type of strength tensor components $R_{1122}$, therefore there is a need for a carefully planned experiments to obtain empirical relationships and justification for $R_{1122}$.Usually, most of the methods of construction based on the limiting surfaces presenting reinforcement material as set anisotropic layers, which in turn causes the study of the properties of the individual layers under load. With the help of the theory of layered media can go from average stress and strain of the composite to the local stresses and strains in any layer. It should be noted that, except for individual works, in all approach ignores voltage and transverse shear deformation $\sigma_{i 3}^{-}, \sigma_{i 3}^{+}(i=1,2)$ and transversal separation or compression $\sigma_{33}^{+}, \quad \sigma_{33}^{-}$.The significant difference between the limiting characteristics of carrier layers and properties of intermediate interfacial layers determines the choice of a particular model of discrete-structural theory of plates and shells. It becomes apparent that the bundle should not be considered as a separate species destruction, and as a factor in determining the form of discrete structural model of multi-layer construction.

Thus, to assess the impact of non-ideal interfacial contact layers criterion (7) should be recorded in a modified form:

$$
\begin{aligned}
& R_{11} \sigma_{11}+R_{22} \sigma_{22}+R_{33} \sigma_{33}+R_{1111} \sigma_{11}^{2}+R_{2222} \sigma_{22}^{2}+R_{3333} \sigma_{33}^{2}+4 R_{1212} \sigma_{12}^{2}+ \\
& +4 R_{1313} \sigma_{13}^{2}+4 R_{2323} \sigma_{23}^{2}+2 R_{1122} \sigma_{11} \sigma_{22}+2 R_{1133} \sigma_{11} \sigma_{33}+2 R_{2233} \sigma_{22} \sigma_{33}=1,
\end{aligned}
$$

Where to strength tensor of a surface (9) by analogy to add additional components:

$$
\begin{gathered}
R_{33}=\frac{\sigma_{33}^{-}-\sigma_{33}^{+}}{\sigma_{33}^{-} \sigma_{33}^{+}} ; R_{3333}=\frac{1}{\sigma_{33}^{-} \sigma_{33}^{+}} ; 4 R_{1313}=\frac{1}{\sigma_{13}^{-} \sigma_{13}^{+}} ; \\
4 R_{2323}=\frac{1}{\sigma_{23}^{-} \sigma_{23}^{+}} ; \\
2 R_{1133}=\frac{R_{11}-R_{33}}{\sigma_{13}^{-}}+R_{1111}+R_{3333}+\frac{1}{\left(\sigma_{13}^{-}\right)^{2}} ; \\
2 R_{2233}=\frac{R_{22}-R_{33}}{\sigma_{23}^{-}}+R_{2222}+R_{3333}+\frac{1}{\left(\sigma_{23}^{-}\right)^{2}}
\end{gathered}
$$

It is assumed that the interlayer shear strength of the material does not depend on the sign of the cross shear stresses, i.e.

$\sigma_{13}^{+}=\sigma_{13}^{-} ; \sigma_{23}^{+}=\sigma_{23}^{-}$. To use a modified criterion (10) it is necessary to experimentally determine the limiting characteristics of layer on the transverse shear and transverse compression or separation.

\section{Results and Discussion}

\subsection{Statement of the Problem and Finding the Stress- Strain State of the Combined Gas Cylinder}

As the object of study selected a combined gas cylinder. First, it was examined fiberglass shell, which consists of a cylindrical portion of length $l=2.163 \mathrm{~m}$ and the radius $R=0.188 \mathrm{~m}$, and bottoms made in the form of hemispheres.

Useful volume of the cylinder is equal to $V=0.2 \mathrm{~m}^{3}$. The shell is made by winding a unidirectional glass tape with the following characteristics: $E_{1}=5.486 \cdot 10^{4} \mathrm{MPa}, E_{2}=1.252 \cdot 10^{4} \mathrm{MPa}$, $E_{3}=1.431 \cdot 10^{4} \mathrm{MPa}, v_{12}=0.058, v_{13}=0.394, v_{23}=0.394$, $G_{12}=3.925 \cdot 10^{3} \mathrm{MPa}, G_{23}=2.683 \cdot 10^{3} \mathrm{MPa}, G_{13}=4.293 \cdot 10^{3} \mathrm{MPa}$. In general, this comprises cylinder twenty unidirectional layers, twelve of which are wound in the circumferential direction, and eight - cross spiral reinforced layer. Winding angle of the spiral layers is $\pm 24^{\circ}$. The thickness of the layer is $\delta=0.0005 \mathrm{~m}$. By the procedure [13] defines the technical constant of the whole package of layers of fiberglass composite material as the one plane of elastic symmetry: 
$E_{1}=2.358 \cdot 10^{4} \mathrm{MPa}, E_{2}=3.747 \cdot 10^{4} \mathrm{MPa}, E_{3}=1.55 \cdot 10^{4} \mathrm{MPa}$, $v_{12}=0.092, v_{13}=0.367, v_{23}=0.352, G_{12}=6.702 \cdot 10^{3} \mathrm{MPa}$, $G_{23}=3.756 \cdot 10^{3} \mathrm{MPa}, G_{13}=3.22 \cdot 10^{3} \mathrm{MPa}$.

The state of stress points inside surface of the bulb when the intensity of the internal pressure $q=20 M P a$. It should be noted that on the borders of the United spherical bottoms and cylindrical shell holds the edge effect. A significant increase in shear stresses transverse shear $\sigma_{13}$ at the points of the middle surface of the cylinder.

In the study of the carrying capacity of the cylinder considered two stages of destruction reinforced shell. In the first stage determines the amount of pressure when there is a destruction of the binder. To do this, use a modified polynomial strength criterion (7), including cross-tangent $\sigma_{\mathrm{i} 3}$ $(i=1,2)$ and transversal stress $\sigma_{33}$.

For this structure fiberglass made the following values of the limiting characteristics of the material strength: $\sigma_{11}^{+}=290 \mathrm{MPa}, \sigma_{22}^{+}=490 \mathrm{MPa}, \sigma_{11}^{-}=200 \mathrm{Ma}, \sigma_{22}^{-}=290 \mathrm{MPa}$, $\sigma_{33}^{+}=50 \mathrm{MPa}, \sigma_{33}^{-}=100 \mathrm{MPa}, \sigma_{12}^{+}=110 \mathrm{MPa}, \sigma_{13}^{+}=\sigma_{23}^{+}=35 \mathrm{MPa}$ Bond breaking occurs at the middle surface at a pressure $q_{1}^{*}=16 \mathrm{MPa}$ further calculation of the bearing capacity was based on the calculation model of the second shell. In the second phase was fixed limit pressure $q_{2}^{*}=23 \mathrm{MPa}$, wherein when failure occurs at the points fiberglass inner surface of the cylindrical shell. In this case, instead of (7), we used the criterion of strength for the case of plane stress at the point (5).

Calculation model of the combined high-pressure cylinder is shown in Fig. 1. We consider an elastic body under the action of internal pressure intensity $q=20 \mathrm{MPa}$. In general, the balloon consists of two parts: the inner part - fiberglass exterior - metal. The inner part of the container carrier is identical previously discussed fiberglass shell. The metal part is made of duralumin B-95: $E=70000 \mathrm{MPa}, v=0.3, \sigma_{B}=500$ $M P a$. The problem is solved in an axisymmetric formulation. The balloon is regarded as a shell of revolution.

To avoid contact of the FRP shell is provided with a metal shell with a minimum size of the gap $h 3=0.002 \mathrm{~m}$, which is filled with an isotropic soft low density material, for example, penovinilplastom with the following technical parameters: $E$ $=83 \mathrm{MPa}, \mathrm{v}=0.33$.

Stress at the inner and outer surfaces of the combined container obtained based on the first calculation model shown in Fig. 3 and 4. $\sigma_{i j}, M P a$.

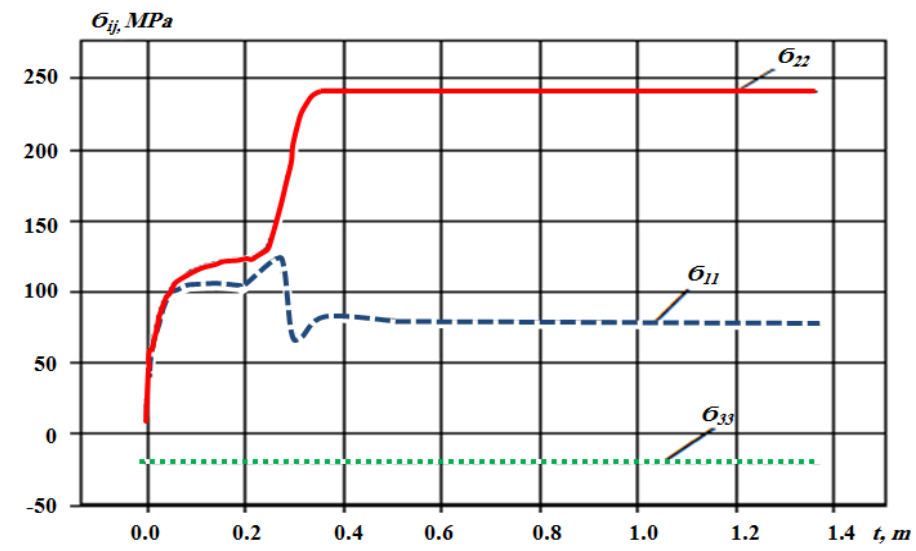

Fig. 3. Changing the meridional $\sigma_{11}$, circumferential $\sigma_{22}$ stresses and stress of transversal compression $\sigma_{33}$ length fiberglass shell (inner surface).

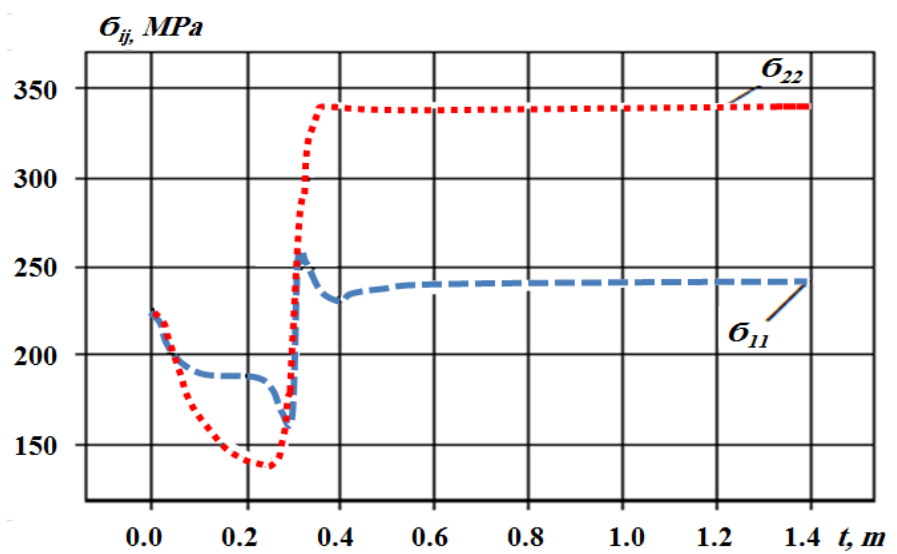

Fig. 4. Changing the meridional $\sigma_{11}$ and circumferential $\sigma_{22}$ stresses along the length of the metallic shell (outer surface).

Deformation fiberglass shell carrier in the longitudinal direction is reduced if an absolute elongation of the FRP shell in the longitudinal direction at a pressure $q=20 \mathrm{MPa}$ was $\Delta l$ $=1.9 \cdot 10^{-2} \mathrm{~m}$, the absolute elongation of the cylinder is equal 
to the combined $\Delta l=0.8 \cdot 10^{-2} \mathrm{~m}$ Reducing the maximum hoop stress fiberglass composite shell cylinder compared to the first embodiment was $40 \%$. With increasing intensity of the internal pressure to $q=30 \mathrm{MPa}$, fiberglass inner shell is almost completely transmits the load on the tougher outer metal shell. Start the destruction of this variant of the combined balloon occurred in the area of the edge effect of the metal plating at a pressure $q^{*}=33 M P a$. To select the optimal design isinvestigated two types of composite cylinders (Table 1). Calculation model of the combined highpressure cylinder is shown in Fig. 2. In general, the balloon consists of two parts: the inner part - fiberglass exterior metal. Combined cylinders mutually equivalent to each other by weight (№ 4 - № 7, Table 1).

Table 1. Two types of cylinder design of composite materials.

\begin{tabular}{|c|c|c|c|c|c|c|}
\hline Number size & $\begin{array}{l}\text { The length of } \\
\text { the cylindrical } \\
\text { portion. } M\end{array}$ & $\begin{array}{l}\text { The outer radius of } \\
\text { the cylinder and } \\
\text { the sphere } R \text {. } M\end{array}$ & $\begin{array}{l}\text { The thickness of } \\
\text { the shell of } \\
\text { fiberglass } h_{C} M\end{array}$ & $\begin{array}{l}\text { The thickness of } \\
\text { the gap } h_{3} \cdot M\end{array}$ & $\begin{array}{l}\text { The thickness of } \\
\text { the plating tank } \\
h_{M} \cdot M\end{array}$ & $\begin{array}{l}\text { Product } \\
\text { Weight.kg }\end{array}$ \\
\hline 1 & 2.163 & 0.188 & 0.0275 & 0 & 0 & 160 \\
\hline $3 *)$ & 0.886 & 0.257 & 0.041 & 0 & 0 & 175 \\
\hline 4 & 2.163 & 0.188 & 0.02 & 0.002 & 0.004 & 154.5 \\
\hline 5 & 2.163 & 0.188 & 0.02 & 0.004 & 0.004 & 160 \\
\hline 7 & 0.886 & 0.257 & 0.03 & 0.004 & 0.006 & 165 \\
\hline
\end{tabular}

*) - Products of KAZAN Experimental Design Bureau "Soyuz" (Certificate of compliance with ISO 9001-2001 № 00107 / RU from 19.01.04g).

The thickness of the fiberglass shell is $h_{1}=0.0275 \mathrm{~m}$; $h_{2}=0.033 \mathrm{~m}$ the first and $h_{3}=0.041 \mathrm{~m}$ second sizes. Technical constant of the whole package of layers of fiberglass composite material as the one plane of elastic symmetry, as well as materials, metal parts and gap filler old. As before, the problem is solved in an axisymmetric formulation. The balloon is regarded as a shell of revolution.

\subsection{Calculations on the Strength of Two Variants of Calculation Models and Comparison of the Optimal Design of a Gas Cylinder}

In the study of the carrying capacity of the cylinder considered two stages of destruction reinforced shell. In the first stage determines the amount of pressure when there is a destruction of the binder. To do this, use a modified polynomial strength criterion, including cross-tangential and transversal stress $\sigma_{33}(7)$.

The coefficients of (7) are determined using the experimentally determined limit of strength $\sigma_{i j}^{+}, \sigma_{i j}^{-}$ $(i, j=1,2,3)$. The index of "+" means that this component the ultimate tensile stress index "-" denotes the ultimate stress in compression. Tensor components for surface strength (7) we have the relations (6) and (8):

It is assumed that the strength of the material on the shift does not depend on the sign of the limit values of shear stresses $\sigma_{12}^{-}=\sigma_{12}^{+}$и поперечных касательных напряжений, т.e. $\sigma_{13}^{+}=\sigma_{13}^{-}, \sigma_{23}^{+}=\sigma_{23}^{-}$. For this structure fiberglass made the following values of the limiting characteristics of the strength of the material: $\sigma_{11}^{+}=290 \mathrm{MPa}, \sigma_{22}^{+}=490 \mathrm{MPa}$, $\sigma_{11}^{-}=250 \mathrm{MPa} \quad, \quad \sigma_{22}^{-}=390 \mathrm{MPa} \quad, \quad \sigma_{33}^{+}=50 \mathrm{MPa}$, $\sigma_{33}^{-}=150 \mathrm{MPa}, \sigma_{12}^{+}=110 \mathrm{MPa}, \sigma_{13}^{+}=\sigma_{23}^{+}=55 \mathrm{MPa}$. Further calculation of the bearing capacity was based on the calculation model of the second shell. The second stage was fixed limit pressure at which failure occurs at the points fiberglass inner surface of the cylindrical shell. In this case, instead of (7), we used the criterion of strength for the case of plane stress at the point (5).

It should be noted that the strength of the material does not depend on the sign of the tangential stress limit value, i.e. $\sigma_{12}^{-}=\sigma_{12}^{+}$. In addition, for an orthotropic material symmetry axes true identity $R_{1112}=R_{2212}=0$.

The calculated value of the internal pressure at which the first phase is destroyed binder fiberglass shell and the second stage the destruction of the whole structure, are shown in Table 2.

Table 2. Shown the calculated value of the internal pressure at which the first phase is destroyed binder fiberglass shell and the second stage the destruction of the whole structure.

\begin{tabular}{llll}
\hline Number size & $\begin{array}{l}\text { Bursting pressure } \\
\text { fiberglass binder } \\
\mathbf{p}_{\mathbf{1}} \text {. MPa }\end{array}$ & $\begin{array}{l}\text { Bursting pressure fiberglass } \\
\text { fibers } \\
\mathbf{p}_{\mathbf{2}} \text {. MPa }\end{array}$ & $\begin{array}{l}\text { Bursting pressure of the } \\
\text { metal plating on III the } \\
\text { theory of strength } \mathbf{p}_{\text {III. }} \text { MPa }\end{array}$ \\
\hline 1 & $27.90 \pm 0.05$ & $58.32 \pm 0.005$ & $\begin{array}{l}\text { Bursting pressure of the } \\
\text { metal plating on IV the } \\
\text { theory of strength } \mathbf{P}_{\text {IV. }} \text { MPa }\end{array}$ \\
2 & $29.45 \pm 0.05$ & $61.93 \pm 0.005$ & -- \\
3 & $28.60 \pm 0.05$ & $64.03 \pm 0.005$ & -- \\
4 & $28.10 \pm 0.05$ & $58.70 \pm 0.005$ & - \\
5 & $27.80 \pm 0.05$ & $55.36 \pm 0.005$ & $42.50 \pm 0.05$ \\
6 & $29.10 \pm 0.05$ & $65.85 \pm 0.005$ & $54.80 \pm 0.05$ \\
7 & $28.60 \pm 0.05$ & $62.07 \pm 0.005$ & $58.05 \pm 0.05$ \\
\hline
\end{tabular}

By varying the stiffness fiberglass shell, as well as by introducing a soft filling between the inside and skin, possible 
to reach conditions equiresistant work material of this design (combined bottle). Deformability carrier fiberglass shell in the longitudinal direction is eliminated fairly rigid along the axis of the cylinder metal protective shell.

Magnitude of the axial elongation are considered types of cylinders in Table 3.

Table 3. Shown magnitudes of the axial elongation are considered types of cylinders.

\begin{tabular}{lll}
\hline \multirow{2}{*}{ Number size } & \multicolumn{2}{l}{ Axial elongation of the balloon $\mathbf{~} \mathbf{~ m m}$} \\
\cline { 2 - 3 } & At an operating pressure of $\mathbf{2 0} \mathbf{~ M P a}$ & At a pressure of bond breaking fiberglass $\mathbf{p}_{\mathbf{1}}$. MPa \\
\hline 1 & 5.96262 & 8.33476 \\
2 & 4.83020 & 7.12792 \\
3 & 3.30364 & 4.72836 \\
4 & 4.80960 & 6.77318 \\
5 & 4.65378 & 6.48584 \\
6 & 2.71664 & 3.95952 \\
7 & 2.60558 & 3.73440 \\
\hline
\end{tabular}

With the increase in the intensity of the pressure inside the shell fiberglass (FRP modulus is three times lower modulus duralumin) almost passes much of the load on the tougher outer metal shell. Start of destruction, the described embodiment of the combined balloon occurred in the area of the edge effect of the metal plating. The theoretical value of the pressure at which the destruction of a metal plating, determined on the basis of the third (the hypothesis of the greatest shear stresses) and fourth (hypothesis Huber-Mises) theory of strength.

Thus, the comparison of fiberglass and composite cylinders (Tables 2 and 3) for their bearing capacity showed that the latter have a number of advantages - they are less deformability, more reliable to use and benefit in terms of thermal insulation properties. Introducing a soft filling between the inside and skin, possible to reach conditions equiresistant work material of this design (combined bottle).

\section{Conclusion}

Comparison of fiberglass and composite cylinders (Table. 2. 3) in their bearing capacity showed that the latter have a number of advantages - they are less deformability. More reliable to use and benefit in terms of thermal insulation properties. Introducing a soft filling between the inner portion and the cladding.it is possible to achieve the same conditions of strong material such this structures (cylinder of composite materials).

\section{Acknowledgment}

This research was supported by Engineering Science Research Program through the Foundation of Technical Education / Technical Institute of Mosul funded by the Ministry of Higher Education and Scientific / Research / Republic of Iraq. (No. 2015-00215).

\section{References}

[1] Gas cylinders - High pressure cylinders for the on-board storage of natural gas as a fuel for automotive vehicles: ISO 11439-2003. - [First edition 2000-09-15]. - ISO. 2003. - 80 p. (International Standard).
[2] High pressure cylinders for compressed natural gas used as a fuel for automotive vehicles. General specifications: GOST R 51753-2001. - [Enacted 29/05/2001]. - M.: GoststandartRussia. 2001. - 20 p. (State Standard of the Russian Federation).

[3] N.V. Pavlov., Creating cars recipients for storage. Transportation and supply of compressed hydrogen/ N.V. Pavlov // Technical gases. - 2008. - № 2. - C. 43-47.

[4] Alexis A. Krikanov., "Composite pressure vessels with higher stiffness" 1999 Elsevier science Ltd. Composite structures. Vol -48. pp 119-127 (2000).

[5] S. Adali. E.B. Summers \& V.E. Verijenko.. "Optimization of Laminated Cylindrical pressure vessels under strength criterion". Composite structures. Vol-25. pp 305-312 (1993). University of national Durban 4001. South Africa.

[6] Tae-Uk Kim..Hvo. Chol Sin., "Optimal design of composite laminated plates with the discreteness in plies angles and uncertain in material properties considered". Computers and Structures 79. pp 2501-2509. (2001).

[7] Levend Parnas., Nuran Katirei., Design of Fiber Reinforced Composite pressure vessels under various loading conditions". Composite structures 58. pp 83-95 (2002).

[8] S.M. Vereshchaka., Nonlinear deformation and stability of multilayer structural elements with structural defects / SM Vereshchaka -Sumy: Publishing House of the SSU. 2009. $286 \mathrm{p}$.

[9] S.K. Godunov., on the numerical solution of boundary value problems for systems of linear ordinary differential equations / S.K. Godunov // Uspekhi Mat. Sciences. - 1961 - 16.№ 3. - S. 171-174.

[10] N.A. Alfutov., Calculation of laminated plates and shells made of composite materials / Alfutov NA, Zinoviev PA, Popov B. G.- M .: Engineering, 1984. - 264 p.

[11] D. Plasticity Drucker and D. Drucker., Inelastic properties of composite materials [ed. K. Gerakovicha; per. with English]. M .: Mir, 1978. - P. 9 - 32

[12] A.K. Malmeyster., Resistance of polymeric and composite materials / Malmeyster A.K. , Tamuzs V.P., Teters G.A. - Riga: Zinatne, 1980. - $572 \mathrm{p}$.

[13] S.M. Vereshchaka., Elastic constants and generalized stiffness of the layered structure of the material / S.M. Vereshchaka // Bulletin of the National the Technical University "KPI". Special Issue: Dynamics and strength of machines. - Kharkov: NTU "KPI". - 2008. - № 36. - S. 29-40. 